\title{
Advanced computational fluid dynamics (CFD)- multi-body simulation (MBS) coupling to assess low-frequency emissions from wind turbines
}

\author{
Levin Klein, Jonas Gude, Florian Wenz, Thorsten Lutz, and Ewald Krämer \\ Institute of Aerodynamics and Gas Dynamics, University of Stuttgart, \\ Pfaffenwaldring 21, 70569 Stuttgart, Germany
}

Correspondence: Levin Klein (levin.klein@iag.uni-stuttgart.de)

Received: 26 June 2018 - Discussion started: 5 July 2018

Revised: 19 September 2018 - Accepted: 23 September 2018 - Published: 17 October 2018

\begin{abstract}
The low-frequency emissions from a generic $5 \mathrm{MW}$ wind turbine are investigated numerically. In order to regard airborne noise and structure-borne noise simultaneously, a process chain is developed. It considers fluid-structure coupling (FSC) of a computational fluid dynamics (CFD) solver and a multi-body simulations (MBSs) solver as well as a Ffowcs-Williams-Hawkings (FW-H) acoustic solver. The approach is applied to a generic $5 \mathrm{MW}$ turbine to get more insight into the sources and mechanisms of low-frequency emissions from wind turbines. For this purpose simulations with increasing complexity in terms of considered components in the CFD model, degrees of freedom in the structural model and inflow in the CFD model are conducted. Consistent with the literature, it is found that aeroacoustic low-frequency emission is dominated by the blade-passing frequency harmonics. In the spectra of the tower base loads, which excite seismic emission, the structural eigenfrequencies become more prominent with increasing complexity of the model. The main source of low-frequency aeroacoustic emissions is the blade-tower interaction, and the contribution of the tower as an acoustic emitter is stronger than the contribution of the rotor. Aerodynamic tower loads also significantly contribute to the external excitation acting on the structure of the wind turbine.
\end{abstract}

\section{Introduction}

Renewable sources of energy and especially wind power have seen a strong expansion in the last years. Even though the construction of large offshore wind farms is currently a strong focus, the potential of onshore wind turbines by opening up new, previously unused areas and repowering of existing sites is still significant. With regard to the acceptance and the fulfillment of stricter legal requirements concerning noise and vibrations, the research on low-frequency emissions from wind turbines gains importance.

\subsection{Emissions from wind turbines}

As wind turbines are counted among the tallest machines on the planet that work in an uncontrolled outside environment, noise and vibration emissions occur in a broad fre- quency range. While sources of acoustic wind turbine emission in the audible range are widely researched and understood and different methods are applied to reduce aerodynamic and mechanical noise (Liu, 2017), much less is known about low-frequency emissions from wind turbines. Many publications about low-frequency emissions from wind turbines concentrate on the impact on seismic measurements. The emitted ground motion signals from wind turbines are measured by local seismic stations built for the detection of events with small magnitudes like far-away earthquakes or nuclear weapons tests. Zieger and Ritter (2018) observed an increase in amplitudes in a frequency range from 0.5 to $10 \mathrm{~Hz}$ dependent on the rotational speed of the turbine and thus wind speed at a distance of $5.5 \mathrm{~km}$ away from a wind turbine. This confirms the measurements by Stammler and Ceranna (2016) and Styles et al. (2005), who found that nearby wind 
turbines reduce the sensitivity of seismic stations as they introduce wind dependence into the measured noise spectra.

Acoustic measurements in the low-frequency range $3.3 \mathrm{~km}$ from a wind farm show discrete peaks at the bladepassing frequency (BPF) and its higher harmonics below $20 \mathrm{~Hz}$ (Hansen et al., 2017). This was also observed by Pilger and Ceranna (2017), who evaluated the data obtained by a micro-barometer array for infrasound detection located in northern Germany. Zajamšek et al. (2016) compared outdoor and indoor measurements close to an Australian wind farm and found the same tonal character in the noise spectra. Hence, the blade-tower interaction is seen to be responsible for aeroacoustic low-frequency noise of wind farms (Van den Berg, 2005).

The scope of research on low-frequency noise from wind turbines is often its impact on human beings. Knopper et al. (2014) conclude from their literature survey that human health is not likely to be affected by low-frequency noise and infrasound from wind turbines. Turnbull et al. (2012) state that the measured level of infrasound within two Australian wind farms was similar to that measured in urban and coastal areas and near other engineered noise sources.

\subsection{Numerical approaches on low-frequency noise}

For an optimization of the structure and foundations of future wind turbines as well as for the assessment of the impact of low-frequency noise and low-frequency seismic vibrations on the environment, reliable methods for the prediction of emissions are of great importance. Gortsas et al. (2017) performed a numerical study to calculate wave propagation using the boundary element method. They developed a model which considers the seismic vibrations mentioned as well as the low-frequency noise in air and even allows a prediction of the sound pressure level (SPL) inside a generic building. But as this model is only capable of calculating the propagation, reliable input data representing the airborne and structure-borne emissions from the wind turbine have to be provided. Computational fluid dynamics (CFD) simulations including fluid-structure interaction (FSI) are capable of providing both. Thus, Gortsas et al. (2017) used data made available by the authors of the present paper.

There are few studies on the modeling of aeroacoustic low-frequency emission from wind turbines. In the 1980s, NASA developed a code for predicting low-frequency wind turbine noise based on Lowson's acoustic equation applied to rotor forces (Viterna, 1981). Madsen (2010) presented a blade element momentum (BEM) based investigation of low-frequency noise that uses the same theory for the aeroacoustic model. CFD simulations combined with the FfowcsWilliams-Hawkings (FW-H) propagation method have been applied by Ghasemian and Nejat (2015) and Bozorgi et al. (2018) to assess low-frequency noise of wind turbine rotors. While Madsen (2010) considered the influence of the tower on the rotor aerodynamics, Ghasemian and Nejat (2015) and
Bozorgi et al. (2018) studied the isolated rotor. Yauwenas et al. (2017) investigated the blade-passage noise of a generic model turbine numerically using CFD and Curle's acoustic analogy. They found a significant contribution of the induced pressure fluctuations on the tower to the tonal blade-passage noise, which was validated with experimental measurements.

In recent years, CFD-based fluid-structure coupling has been applied frequently for the investigation of wind turbines. Li et al. (2017) presented a framework of a wind turbine aero-servo-elastic simulation including flexible blades and tower which allows the motion of all turbine components. In their approach, controllers for torque and blade pitch are included as well, and they focus their studies on the impact of FSI on aerodynamic rotor loads, drive train dynamics, controllers and wake. Streiner et al. (2008) developed a coupling of the CFD code FLOWer to the multi-body simulations (MBS) solver SIMPACK with the capability to couple isolated wind turbine rotors.

\subsection{Scope and objectives}

A totally revised FLOWer-SIMPACK coupling is revealed in the present paper with the potential to take into account more degrees of freedom, like tower deformation or changes in rotational speed in the structural model and their impact on aerodynamics and aeroacoustics, respectively. Together with the already existing process chain, fully coupled CFD simulations under realistic turbulent inflow conditions can be conducted, providing both airborne and structure-borne emissions simultaneously. A FW-H in-house code is applied to calculate acoustic pressure at distant observers while tower base loads represent the structure-borne emission. The aim of the present paper is to identify the sources of low-frequency emissions and to investigate the impact of the complexity of the numerical model on the calculated low-frequency emissions from a generic $5 \mathrm{MW}$ wind turbine. The complexity of the model is increased from a rotor only simulation with uniform inflow to a coupled simulation including blade, tower and foundation dynamics with turbulent atmospheric boundary layer. The spectra of tower base loads and SPLs for seven cases overall are compared in a frequency range from 0.1 to $25 \mathrm{~Hz}$ for evaluation.

\section{Numerical process chain}

A high-fidelity process chain based on multiple solvers is established for the investigation of low-frequency emissions from wind turbines. It consists of the CFD solver FLOWer, the MBS solver SIMPACK and the FW-H solver ACCO. A coupling between FLOWer and SIMPACK was developed to generate high-fidelity time series of surface pressure distribution on the turbine and structural loads (forces and moments) acting on the foundation of the turbine. Using the CFD results, the aeroacoustic signal at distant, predefined observer positions is computed by means of ACCO. 


\subsection{CFD solver}

FLOWer is a compressible, dual time-stepping, block structured Reynolds-averaged Navier-Stokes (RANS) solver developed by the German Aerospace Center (DLR) (Kroll et al., 2000). The use of independent grids for bodies and background is enabled by the overlapping grid technique CHIMERA, one of FLOWers' main features. The solver is continuously extended at the Institute of Aerodynamic and Gas Dynamics (IAG) regarding functionality and performance, including, amongst others, the higherorder finite-difference weighted essentially non-oscillatory (WENO) scheme (Kowarsch et al., 2013), Dirichlet boundary condition to apply arbitrary unsteady inflow, a body forces approach to superimpose turbulence (Schulz et al., 2016b) and various detached-eddy simulation (DES) schemes (Weihing et al., 2018). The capability of FLOWer for wind turbine simulations has been shown in several projects. The interaction of a wind turbine in complex terrain with atmospheric turbulence was investigated by Schulz et al. (2016a) and code to code comparisons were recently conducted in the European AVATAR project (Schepers et al., 2016).

\subsection{MBS solver}

SIMPACK is a commercial nonlinear MBS solver that can be applied to simulate dynamic systems consisting of rigid and flexible bodies. Flexible turbine components like tower and blades are modeled with linear or nonlinear beam theory. The kinematics between the components are defined by joint elements and internal forces can be considered. There are two ways to apply external forces such as aerodynamic forces: either by built-in interfaces or by programmable user routines. Controllers can also be integrated. SIMPACK has been recently applied by industry and research groups for the simulation of wind turbines. Examples can be found in Luhmann et al. (2017) and Jassmann et al. (2014).

\subsection{Fluid-structure interaction}

To take the influence of unsteady structural deformation on the aerodynamics into account, a revised coupling between FLOWer and SIMPACK is implemented. The new approach generally allows the coupling of slender beam-like structures and is not limited to rotor blades or even wind turbines. Combined coupling of rotating and non-rotating parts can be applied and the deformation of adjacent structures is considered. Furthermore, coupling is not restricted to flexible deformations but rigid-body motions (rotations and translations) can also be realized. In the application to wind turbines, pitch motions and changes in rotational speed of the rotor can be transferred from the MBS solver to the CFD solver.

For the technical realization, an existing interface that was developed to couple SIMPACK with the fluid solver ANSYS CFX for the investigation of a tidal current turbine (Arnold et al., 2013) is extended. Furthermore, libraries for grid deformation and load integration which have recently been developed and integrated into FLOWer (Schuff et al., 2014; Kranzinger et al., 2016) are extended for the coupling with SIMPACK. Without restrictions in functionality, the setup of the coupling is kept simple and the dependencies between MBS and CFD models are low. Thus, the resolutions of the CFD and MBS models are independent of each other, which allows a fast and easy adjustment and replacement of MBS structures or CFD meshes. Furthermore, the new coupling can be restarted, allowing much longer simulation times if FLOWer runs on clusters with limited job duration. It was already successfully applied to the blade of a generic $10 \mathrm{MW}$ turbine for comparison reasons by Sayed et al. (2016), who implemented a coupling of FLOWer to the structural dynamics solver Carat++.

\subsubsection{General functionality}

The developed coupling is a partitioned approach, where two independent solvers run simultaneously on different machines and exchange data via a Secure Shell (SSH) connection at discrete positions, so-called markers. The markers are positioned inside the bodies. While rigid bodies only have one marker, flexible bodies like rotor blades have several markers that are distributed along the beam. On the one hand, deflections and rotations of these markers relative to their non-deformed position are computed by SIMPACK. On the other hand, aerodynamic forces and moments acting on these markers are calculated in FLOWer. For each structure that is coupled, a communication coordinate system is defined that has to be in the same position and same orientation in both models at all times. It does not have to be fixed but can be rotating or translating in a predefined way. All data concerning the respective structure is communicated in this coordinate system.

\subsubsection{Mesh deformation}

The task of the deformation library implemented in FLOWer is to apply the deformations of the markers on the corresponding CFD surfaces and to deform the surrounding volume mesh accordingly. The surface is represented by a point cloud which is generated from the CFD mesh. For rigid structures only one marker is used and all surface cloud points perform a rigid-body motion based on the translation and rotation of this marker. A cubic spline interpolation is applied for the mapping of flexible structures (beams) consisting of more than one marker. The deformation of each surface cloud point is then realized as rigid-body motion based on the corresponding positions along the beam. While a complete spline approach is used for the deflections, taking the rotation at the end points into account, the rotations and the non-deformed marker positions are interpolated using natural splines. A similar approach has been presented by Arnold et al. (2013). 


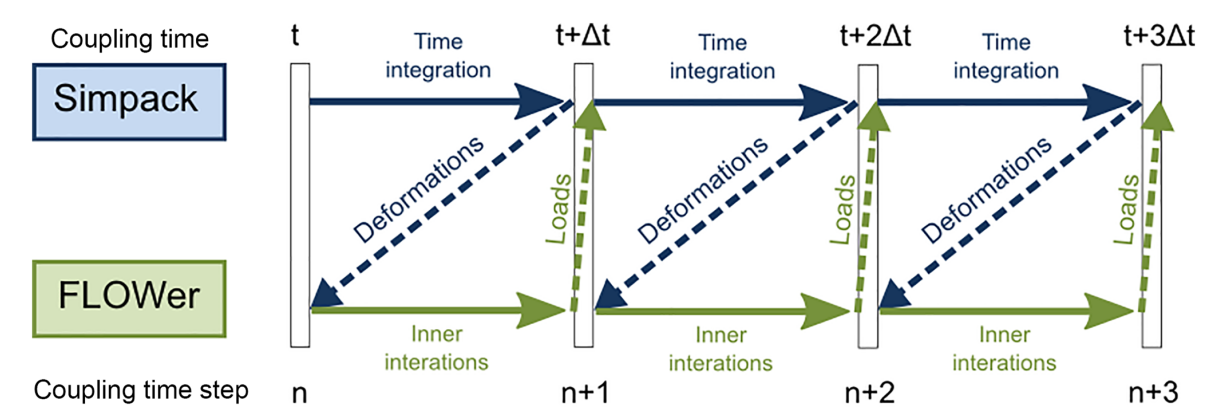

Figure 1. Explicit coupling scheme of the FLOWer-SIMPACK coupling.

Finally, the volume grids are deformed based on the deformation of the point cloud using radial basis functions. To ensure correct overlapping of deformed meshes, holes associated with the deformed surface can also be deformed.

\subsubsection{Load integration}

The load library implemented in FLOWer enables the calculation of aerodynamic loads on grid surfaces by the integration of friction and pressure over the cell faces. This is also necessary for the coupling to SIMPACK, as there is no surface in the structural model and the aerodynamic forces have to be mapped to the discrete marker positions. For this purpose, the CFD surface is divided into segments based on the deformed marker positions. For each of these segments, loads are integrated and afterwards assigned to the respective markers. Moments are calculated with respect to the origin of the corresponding communication coordinate system. For structures with only one marker, loads are integrated over the whole CFD surface of the respective structure.

\subsubsection{Communication interface}

The communication is realized by means of files. Data files contain deformations or loads and status files indicate that the data file is ready to be read. While SIMPACK is running on a local Windows machine, FLOWer is usually executed in parallel mode on a high-performance computing (HPC) system running on Linux. A portable communication script in Windows' inherent scripting language PowerShell enables fast and reliable communication between the two solvers. The Linux machine is accessed using a SSH connection via the Windows Secure Copy (WinSCP) client.

\subsubsection{Coupling scheme}

In the presented work, an explicit coupling scheme is applied. The size of the coupling time step is equal to the physical FLOWer time step and remains constant throughout the simulation. Both solvers run in a sequential way, waiting for the other solver to reach the next time step and to send communication data. SIMPACK runs one time step ahead doing time integration with the aerodynamic loads that FLOWer computed at the end of the previous time step (Fig. 1).

\subsection{Acoustic solver}

Acoustic pressure at arbitrary observer locations is calculated by means of the in-house FW-H solver ACCO. Pressure and velocities on surfaces enclosing the noise sources are evaluated at each time step of the transient CFD solution, including velocities due to the deformation, translation and rotation. For the present study, the surfaces used for the acoustic analysis are identical with the physical surfaces of the turbine (rotor, tower, hub, etc.). Volume sources generated by free-flow turbulence are neglected, which is justified for low Mach number flow because quadrupole volume noise is proportional to $\mathrm{Ma}^{7}$. This approach was validated for a rod-cylinder configuration and an airfoil in turbulent flow (Lutz et al., 2015; Illg et al., 2015). The acoustic monopole and dipole contributions to the observer sound pressure level (SPL) are computed by means of the FW-H equation. Its left-hand side is the wave equation which describes the transmission of sound to the observer, presuming undisturbed propagation and observers located in the acoustic far field. Hence, ground reflections and nonlinear propagation due to atmospheric layering and turbulence are not taken into account. The acoustic far field is defined by the presence of a fully developed wave front and thus starts several wave lengths away from the source. Parallel execution of ACCO allows the computation of noise carpets consisting of several thousand observer locations.

The application of the FW-H analogy allows the evaluation of the contribution of selected components of the wind turbine to SPL by excluding surfaces of particular components (e.g., tower) from the analysis.

\subsection{Computational setup}

\subsubsection{The turbine}

The examined turbine is based on the generic $5 \mathrm{MW}$ turbine developed by the National Renewable Energy Laboratory (NREL; Jonkman et al., 2009) and was slightly modified 


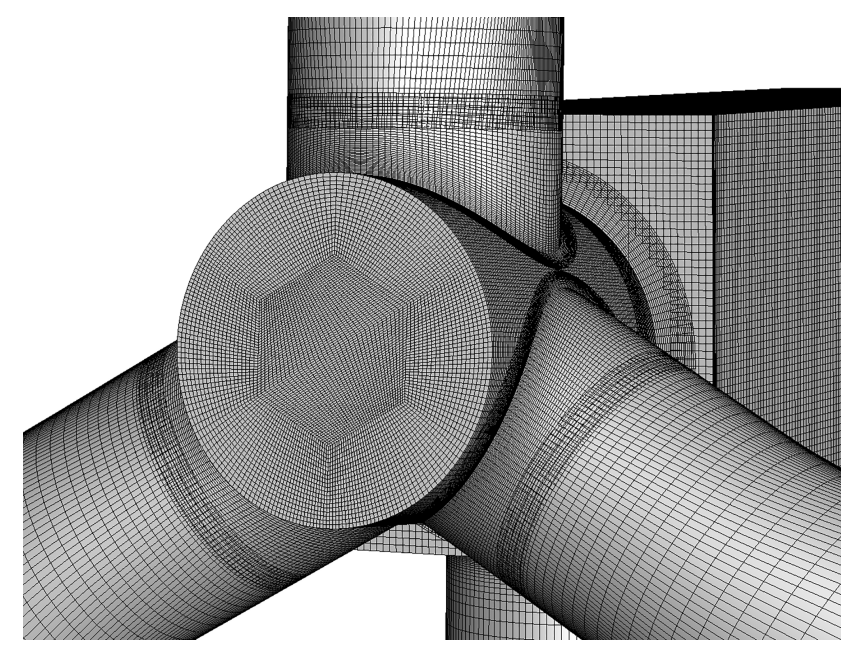

Figure 2. CFD surface mesh, showing the connection of hub, blades and nacelle with overlapping meshes.

in the OFFWINDTECH project (Bekiropoulos et al., 2013). The main modifications concern the rated conditions which were changed to a rotational speed of 11.7 RPM and a pitch angle of $-2.29^{\circ}$ at a wind speed at hub height of $11.3 \mathrm{~m} \mathrm{~s}^{-1}$. The turbine is investigated at rated conditions in an onshore configuration with a hub height of $90 \mathrm{~m}$, a rotor diameter of $126 \mathrm{~m}$ with a tilt angle of $5^{\circ}$ and a pre-cone angle of $2.5^{\circ}$. The original tower with a bottom diameter of $6 \mathrm{~m}$ and a top diameter of $3.87 \mathrm{~m}$ is used.

\subsubsection{CFD model}

The CFD model of the OFFWINDTECH turbine consists of 10 independent body meshes, which are embedded in a Cartesian hanging grid node background mesh using the CHIMERA technique. Blades, hub, nacelle and tower are considered in the simulation with fully resolved boundary layer $\left(y^{+} \leq 1\right)$. No gaps are left between the components of the turbine, as blade-hub connectors and a hub-nacelle connector are included in the CFD mesh (Fig. 2). Blades are meshed in a $\mathrm{C}-\mathrm{H}$ mesh topology with 120 cells in radial direction and 180 cells around the airfoil, summing up to approximately 5.3 million cells per blade. Two different Cartesian background grids with hanging grid nodes are used. One for the case with prescribed atmospheric turbulence where the mesh is additionally refined to a cell size of $1 \mathrm{~m}^{3}$ upstream of the turbine (64.5 million cells) and another for the case without atmospheric turbulence where only the mesh close to the turbine is refined (20.8 million cells). The computational domain is approximately 48.8 rotor radii $(R)$ long (12.7 $R$ upstream of the rotor plane), is approximately $24.4 R$ wide and has a height of approximately $16.2 R$. According to a previous study using FLOWer (Sayed et al., 2015), the background grids expand more than sufficiently in all directions to avoid influencing the flow field around the turbine.
Table 1. Details on the foundation of the wind turbine, similar to Gortsas et al. (2017).

\begin{tabular}{ll}
\hline Mass & $1.888 \times 10^{6} \mathrm{~kg}^{2}$ \\
Inertia $x, y$ & $82.705 \times 10^{6} \mathrm{~kg} \mathrm{~m}^{2}$ \\
Inertia $z$ & $88.529 \times 10^{6} \mathrm{~kg} \mathrm{~m}^{2}$ \\
Stiffness $x, y$ & $8.554 \times 10^{9} \mathrm{~N} \mathrm{~m}^{-1}$ \\
Stiffness $z$ & $7.332 \times 10^{9} \mathrm{~N} \mathrm{~m}^{-1}$ \\
Rotational stiffness $x, y$ & $559 \times 10^{9} \mathrm{Nm} \mathrm{rad}^{-1}$ \\
Rotational stiffness $z$ & $559 \times 10^{9} \mathrm{Nm} \mathrm{rad}^{-1}$ \\
Damping $x, y$ & $240 \times 10^{6} \mathrm{Ns} \mathrm{m}^{-1}$ \\
Damping $z$ & $325 \times 10^{6} \mathrm{Ns} \mathrm{m}^{-1}$ \\
Rotational damping $x, y$ & $5.035 \times 10^{9} \mathrm{Nm} \mathrm{sad}^{-1}$ \\
Rotational damping $z$ & $4.180 \times 10^{9} \mathrm{Nm} \mathrm{sad}^{-1}$ \\
\hline
\end{tabular}

Overall, the fine setup consists of 86 million cells and the coarse setup of 42 million cells.

Concerning inflow, three different cases are regarded in the present study. Uniform inflow, steady atmospheric boundary layer and turbulent atmospheric boundary layer. An exponent of 0.19 is applied for the power law profile describing the steady atmospheric boundary layer, keeping the wind speed at hub height at $11.3 \mathrm{~ms}^{-1}$. Atmospheric turbulence with a reference length scale of $42 \mathrm{~m}$, created using Mann's model (Mann, 1994), is introduced into the flow field using body forces $16 \mathrm{~m}$ downstream of the inlet, superimposing the steady boundary layer profile. The resulting turbulence level at the turbine position is $16 \%$. Unsteady RANS (URANS) simulations are applied with a secondorder dual time-stepping scheme for temporal discretization. The second-order central discretization with the JamesonSchmidt-Turkel (JST) artificial dissipation term is used for spatial discretization in body meshes, and the fifth-order WENO scheme is applied to the background mesh in order to reduce the dissipation of vortices. Menter's shear stress transport (SST) model (Menter, 1994) is deployed for turbulence modeling. A physical time step corresponding to $0.75^{\circ}$ azimuth $(\approx 0.01068 \mathrm{~s})$ with 100 inner iterations is applied for the evaluated part of the simulations.

\subsubsection{Structural model}

The SIMPACK model of the OFFWINDTECH turbine was built by Matha et al. (2010). The blades are modeled nonlinearly by using multiple flexible bodies per blade. The structural properties of the tower are adopted from the NREL $5 \mathrm{MW}$ turbine (Jonkman et al., 2009) taking 20 modes into account. Hub and nacelle are defined as rigid bodies. The foundation is modeled as a rigid body connected to the ground with a spring-damper system. Details can be found in Table 1. 
Table 2. Definition of simulation cases, ordered according to increasing complexity.

\begin{tabular}{lllll}
\hline Case name & Inflow & CFD structures & Flexible structures & Background mesh \\
\hline LC1 & uniform & rotor & none & coarse \\
LC2 & uniform & rotor, nacelle, tower & none & coarse \\
LC2_FSC1SD & uniform & rotor, nacelle, tower & rotor blades SD & coarse \\
LC2_FSC1 & uniform & rotor, nacelle, tower & rotor blades & coarse \\
LC2_FSC3 & uniform & rotor, nacelle, tower & rotor blades, tower, foundation & coarse \\
LC3_FSC3 & steady ABL & rotor, nacelle, tower & rotor blades, tower, foundation & fine \\
LC4_FSC3 & turbulent ABL & rotor, nacelle, tower & rotor blades, tower, foundation & fine \\
\hline
\end{tabular}

ABL, atmospheric boundary layer; SD, steady deformation.

\subsubsection{FSI setup}

Overall, 160 markers are used for the fluid structure coupling of the OFFWINDTECH turbine (Fig. 3), 49 markers for each blade, 11 markers for the tower, and 1 marker each for the nacelle and hub. Since in the structural model and the CFD model a fixed rotational speed is prescribed, a rotating communication coordinate system in the center of the hub is used for the rotating parts. The communication for tower and nacelle is performed in a fixed coordinate system placed at the tower base (Fig. 3). In the SIMPACK model of the turbine, additional rigid bodies are created for the definition of the undeformed markers. The corresponding moving markers are attached to the flexible structures of the turbine. With this approach, the measured deformations between deformed and undeformed markers are composed of flexible deformations of the body itself plus rigid-body motion due to the deformation or motion of the adjacent body.

\subsubsection{Simulation cases}

In Table 2 all regarded simulation cases are listed. For evaluation they are assigned to three studies. In the first study, no FSI is considered and thus all turbine components are kept rigid. The influences of the presence of the tower and the distance of the blade to the tower are evaluated at uniform inflow conditions by comparing LC1, LC2 and LC2_FSC1SD. In case LC2_FSC1SD, the blade deformation is equal to the averaged blade deformation of case LC2_FSC1 to obtain a realistically deformed shape of the blades with reduced distance between blades and tower. In a second study the degrees of freedom of the structural model are increased at uniform inflow conditions. Three cases are compared: the rigid case with steady deformed blades (LC2_FSC1SD), a case with flexible blades (LC2_FSC1), and a case with flexible blades as well as a flexible tower and foundation (LC2_FSC3). In the third study, the inflow conditions are varied, keeping the structural model the same. Case LC2_FSC3 is used as reference. A steady atmospheric boundary layer (ABL) is prescribed at the inlet by means of a power law inflow profile in case LC3_FSC3. This steady ABL is superposed with veloc-

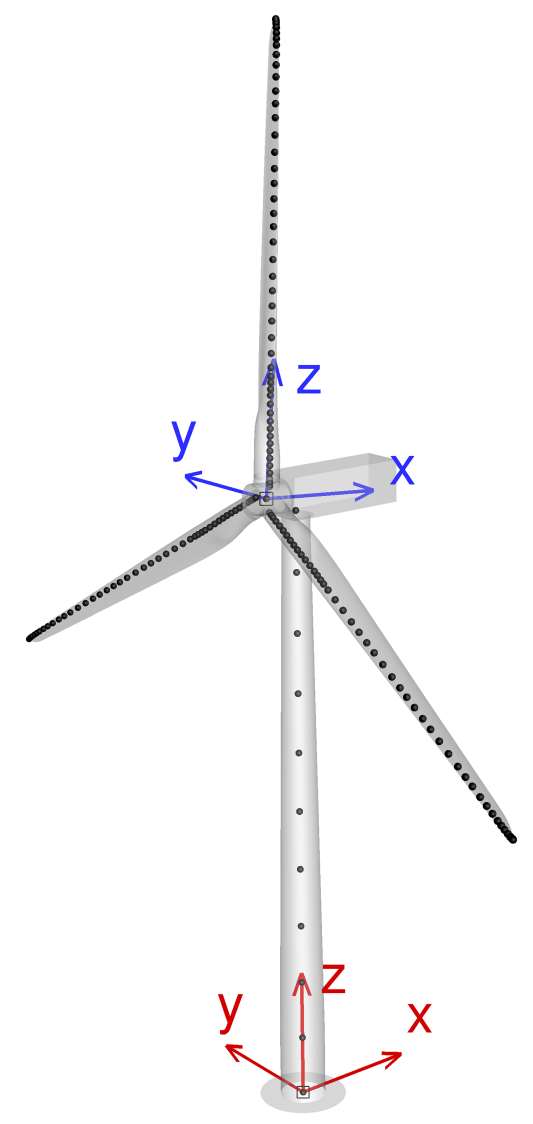

Figure 3. CFD surface of turbine including markers for coupling with SIMPACK. Rotating hub coordinate system is shown in blue and tower base coordinate system in red.

ity fluctuations modeling a turbulent atmospheric boundary layer in the case of LC4_FSC3.

\subsection{Evaluation}

The aim of the simulation chain is to model airborne and structure-borne emissions simultaneously by evaluating SLPs at distant observers and load fluctuations at the tower base. In the fluid-structure coupled simulations tower base 


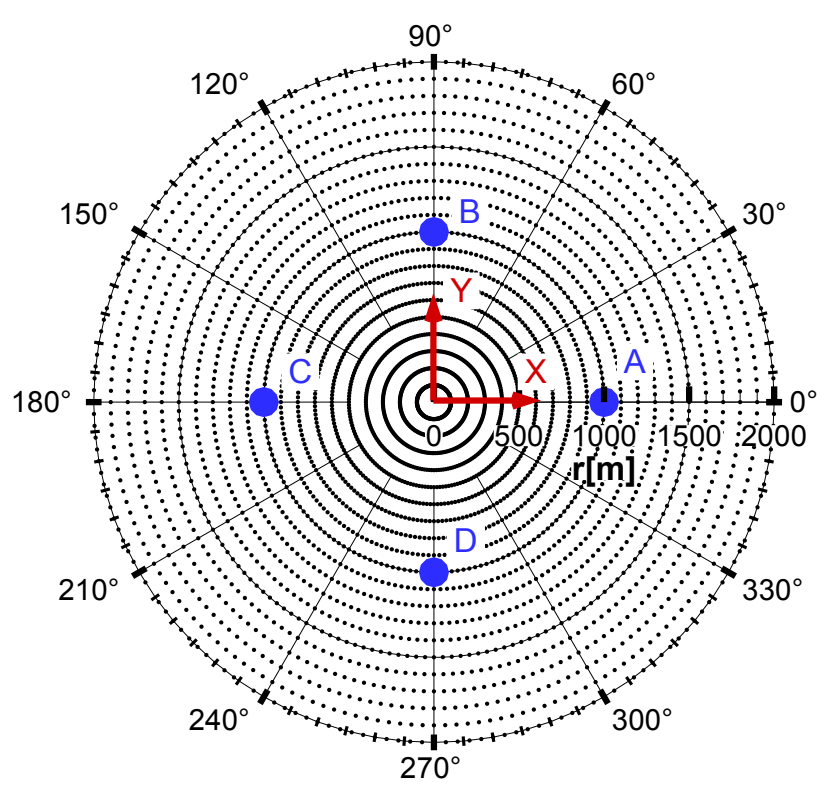

Figure 4. Observer positions for the evaluation of aeroacoustic emissions. Tower base coordinate system shown in red. View from above; turbine in the center; wind from left.

loads are evaluated directly in the structural model at the interface between tower and foundation, whereas in the noncoupled simulations aerodynamic loads are computed from CFD results. In both cases the tower base loads are presented with respect to the tower base coordinate system which is shown in Fig. 3. The temporal resolution of the data is equal to the coupling time step. To achieve the same temporal resolution in the acoustic emission, each time step a CFD surface solution is saved temporally as input for the acoustic simulations.

Acoustic simulations using ACCO are conducted to calculate the acoustic pressure at a carpet of observers on the ground surrounding the turbine. Figure 4 shows the 3600 observers located on 20 concentric rings around the turbine at radial positions of 100 to $2000 \mathrm{~m}$ with a radial resolution of $100 \mathrm{~m}$ and a circumferential resolution of $2^{\circ}$. Unweighted SPL is calculated from sound pressure time series at the observers with a reference sound pressure of $20 \mu \mathrm{Pa}$. The sound propagation and directivity for discrete frequencies can be evaluated by plotting the SPL contour on the ground. Four observers at a distance of $1000 \mathrm{~m}$ from the turbine are chosen for a detailed evaluation of SPL spectra (large dots in Fig. 4). Prior to frequency analyses by means of fast Fourier transform (FFT), the time series signals of loads and sound pressure are cut to multiples of one rotational period of the turbine in order to supply a preferably periodical signal to the FFT and to avoid the influence of start-up effects. In coupled simulations, the first two revolutions are excluded from evaluation. For case LC4_FSC3 14 revolutions and for all other cases, eight revolutions are evaluated. As the sampling rate is (a)

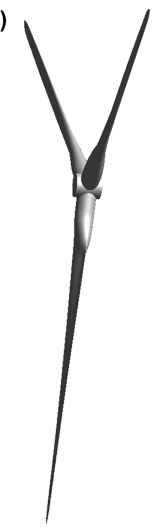

(b)

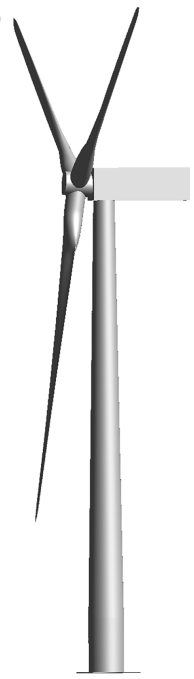

(c)

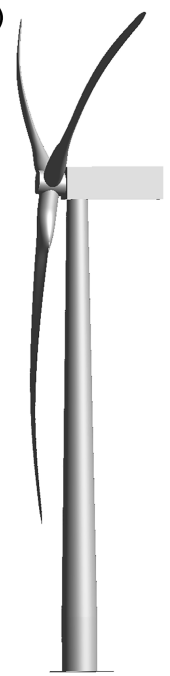

Figure 5. CFD turbine surfaces of cases LC1 (a), LC2 (b) and LC2_FSC1SD (c). Snapshot with one blade in front of the tower at $180^{\circ}$ azimuth.

equal to the physical time step of the simulation, the highest resolved frequency (Nyquist frequency) is $46.8 \mathrm{~Hz}$.

\section{Results}

\subsection{Rigid simulations}

In this section three non-fluid-structure coupled cases are compared at uniform inflow conditions. As reference the rotor only case (LC1) is regarded where unsteady effects on the loads only result from the tilt of the rotor, the proximity to the ground and unsteady flow separation. In a second case, the tower is considered (LC2), and in a third case steady deformation is applied to the blades (LC2_FSC1SD). The CFD surfaces of all three cases are shown in Fig. 5.

\subsubsection{Tower base loads}

In the non-fluid-structure coupled cases, no unsteady structural forces occur as all structures are rigid. Thus, load fluctuation only arises from aerodynamics. Figure 6 shows the spectra of $M_{x}$ and $M_{y}$ of all three cases with respect to the tower base coordinate system (moment reference point). No distinctive peaks can be found in the spectra of LC1. After including the tower in the simulation (LC2), sharp peaks at the blade-passing frequency and its higher harmonics appear with significantly increased amplitudes up to a frequency of approximately $10 \mathrm{~Hz}$. Regarding $M_{x}$, a general increase in the amplitudes below BPF is present with a peak at approximately $0.3 \mathrm{~Hz}$ caused by vortex shedding, which will be shown later. In LC2_FSC2SD the distance between tower and blades is reduced due to the steady deformation of the blades. This leads to an increase in the amplitudes 

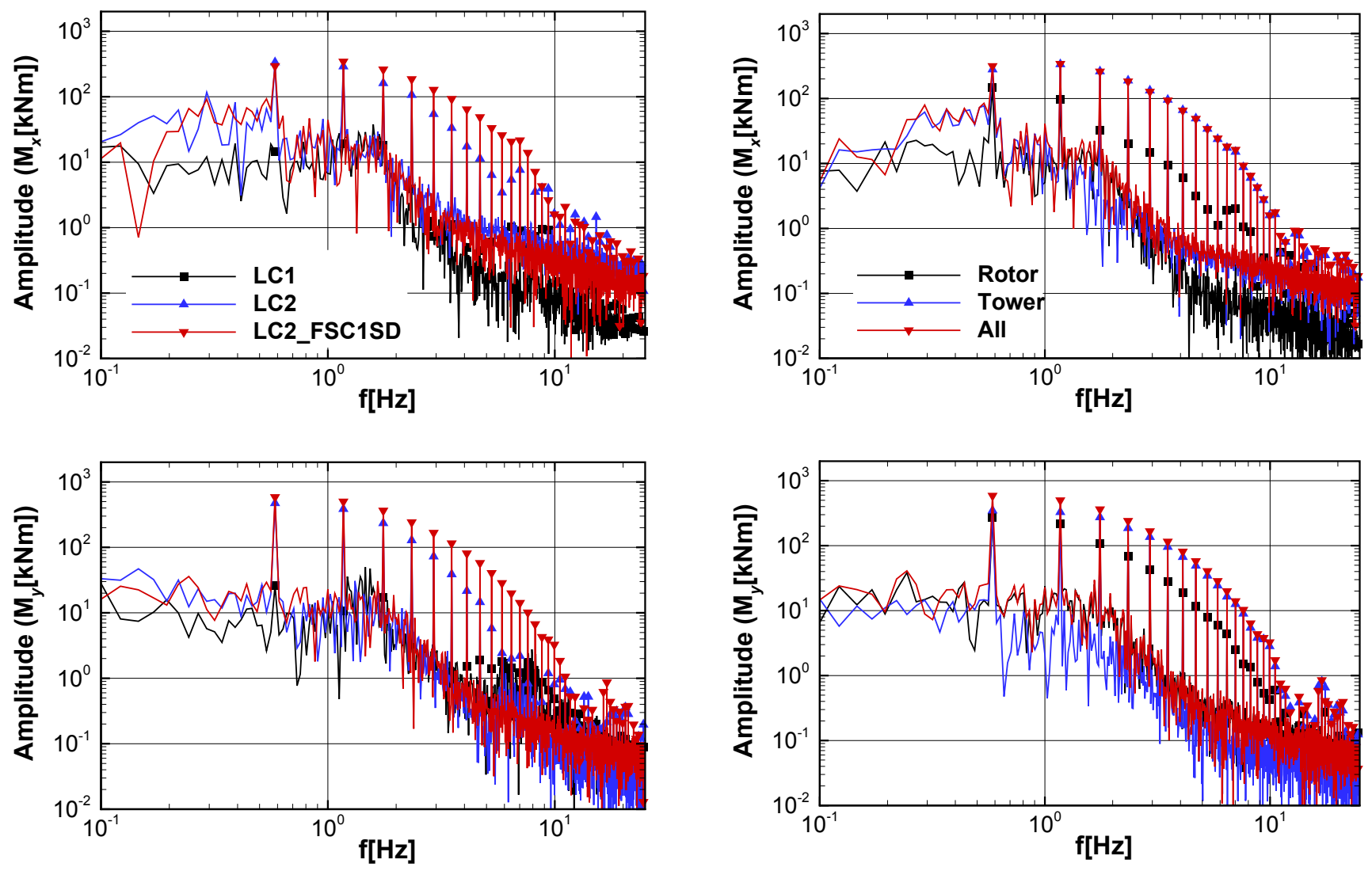

Figure 6. Spectra of aerodynamic loads with respect to tower base (moment reference point) for cases LC1, LC2 and LC2_FSC1SD.

at blade-passing harmonics. The relative increase is stronger for higher frequencies. The amplitude of $M_{y}$ is increased by more than $50 \%$ for frequencies between 5 and $10 \mathrm{~Hz}$. For $M_{x}$ the amplitude at BPF stays almost constant while amplitudes are increased for the higher harmonics compared to case LC2. The maximum amplitude of $M_{x}$ is shifted to the second harmonic of BPF. The amplitudes of $M_{z}$ are much lower compared to the other load components and are therefore not shown.

The composition of the aerodynamic loads is investigated in detail for case LC2_FSC1SD. Therefore, aerodynamic loads on rotor and tower were evaluated separately with respect to the tower base coordinate system (moment reference point). Figure 7 shows the resulting spectra. The peak amplitudes of the tower spectra are dominant over the whole frequency range. Especially for $M_{x}$, the tower load amplitudes are up to 10 times higher compared to the rotor load amplitudes. For $M_{x}$ the general level below BPF is higher in the tower load spectra. This can be interpreted as the impact of unsteady flow separation at the tower induced by vortex shedding. This phenomenon, known as von Kármán vortex street, leads to unsteady forces on blunt bodies with a frequency described by the dimensionless Strouhal number. Assuming an

inflow velocity of $8 \mathrm{~m} \mathrm{~s}^{-1}$ (reduced due to induction of the rotor) results in a Reynolds number of $2.8 \times 10^{6}$ with respect to the mean diameter of the tower $(4.9 \mathrm{~m})$. The corresponding Strouhal number of approximately 0.24 leads to a theoretical vortex shedding frequency of $0.38 \mathrm{~Hz}$. As both diameter and inflow velocity are not constant over the length of the tower and inflow is disturbed by the rotor, a broader range of vortex shedding frequencies can be expected as it is present in the spectrum of $M_{x}$.

The surface pressure amplitudes on the tower are displayed in Fig. 8 at two different frequencies. At BPF $(0.585 \mathrm{~Hz})$ as well as at $0.292 \mathrm{~Hz}$ where the spectra of $M_{x}$ have a local maximum. A strong peak appears at BPF at the front of the tower shifted to the side of the approaching blade. The symmetric shape of the pressure amplitude distribution and the higher amplitudes at the rear side of the tower at $0.292 \mathrm{~Hz}$ can very likely be associated with vortex shedding creating the peak in the load spectra. These observations support the idea of the superposition of blade-passing effects and vortex shedding at the tower. 
(a)

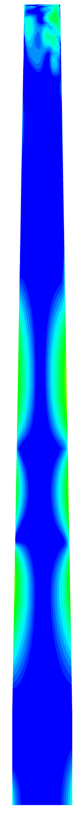

Front

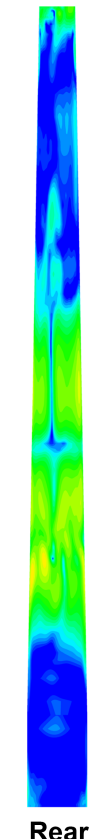

$0.292 \mathrm{~Hz}$ (b)

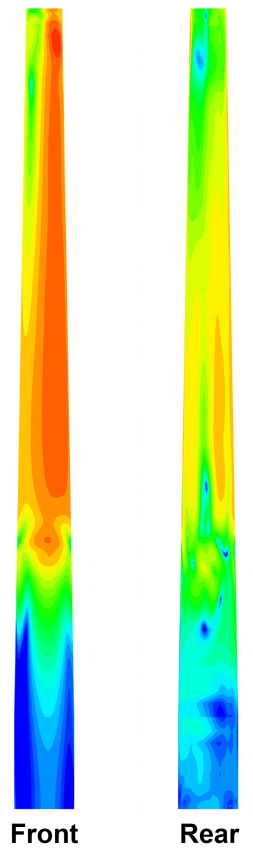

$0.585 \mathrm{~Hz}$

Amplitude (pressure[Pa]): 1

10

100

Figure 8. Pressure amplitudes on CFD tower surface of case LC2_FSC1SD at $0.292 \mathrm{~Hz}$ (a) and blade-passing frequency $(0.585 \mathrm{~Hz})(\mathbf{b})$.

\subsubsection{Aeroacoustic emission}

Figure 9 shows the spectra of the SPL for observers $C$ and $\mathrm{D}$ for the cases LC1, LC2 and LC2_FSC1SD. The spectrum at observer $\mathrm{A}$ is very similar to the one at observer $\mathrm{C}$. The same applies to observers B and D. The maximum SPL for $\mathrm{LC} 1$, the case without a tower, occurs at observer B at BPF and is the only prominent peak. The emission at this frequency shows a strong directivity, as the amplitude is much higher at the sides than upstream and downstream of the turbine. The presence of the tower (LC2 and LC2_FSC1SD) causes a massive increase in amplitudes at the BPF harmonics while the broadband noise level stays low. The highest peak appears upstream of the turbine at observer $C$ at the third BPF harmonic and is approximately $4 \mathrm{~dB}$ higher in case LC2_FSC1SD compared to case LC2. The spectra of case LC2 show only a weak directivity for the BPF harmonics as the amplitudes at the upstream and downstream observers are just slightly lower than at the side observers. A stronger directivity can be observed for case LC2_FSC1SD at BPF where the amplitudes are clearly higher at the upstream and downstream observer. Compared to case LC1 the SPL at frequencies below BPF also rises but only at observer positions B and D. Comparing LC2_FSC1SD to LC2, the increase in amplitudes due to reduced blade-tower distance is
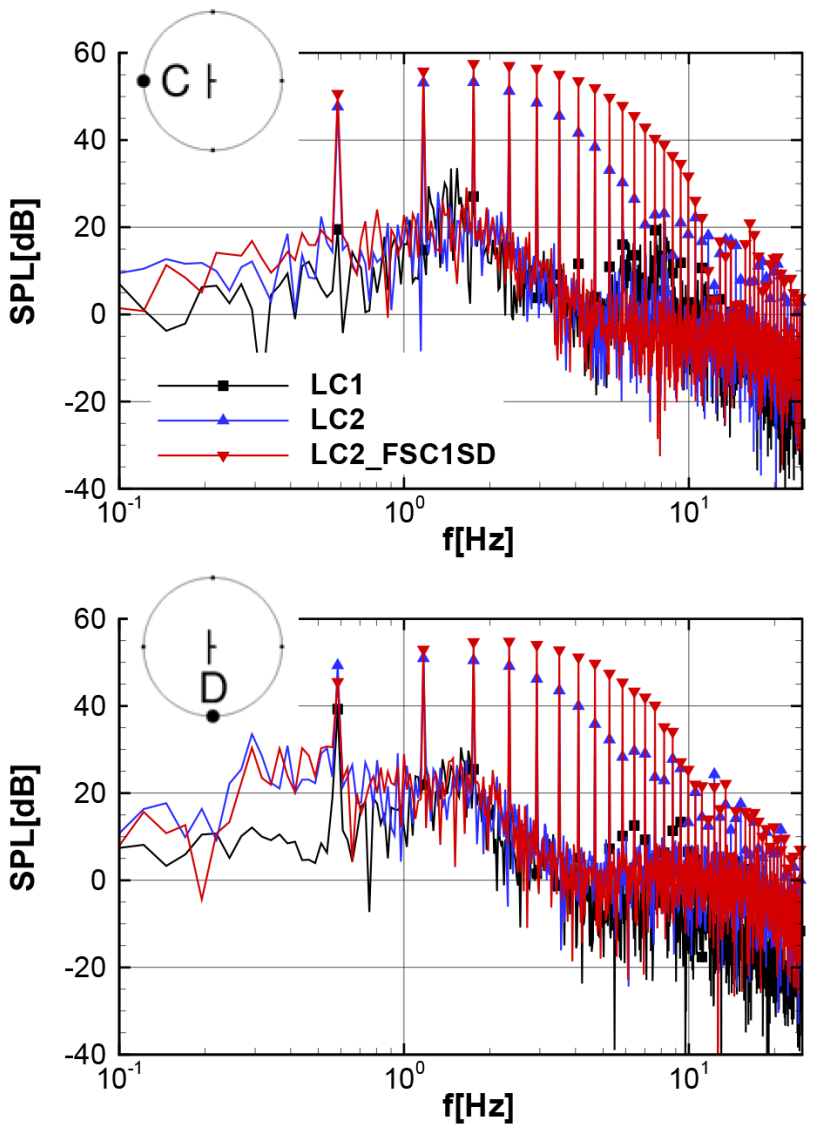

Figure 9. Spectra of unweighted SPL (reference sound pressure of $20 \mu \mathrm{Pa}$ ) at two observer positions on the ground with a distance of $1000 \mathrm{~m}$ from the turbine for cases LC1, LC2 and LC2_FSC1SD.

most prominent between the 5th and 10th harmonic of BPF where it amounts to more than $10 \mathrm{~dB}$. The SPL peaks drop below $20 \mathrm{~dB}$ at around $15 \mathrm{~Hz}$ even for case LC2_FSC1SD.

To examine the aeroacoustic noise emission in detail, the noise emission originating from tower and rotor surfaces are evaluated separately for case LC2_FSC1SD. Figure 10 shows the SPL spectra at observer positions C and D. It can be seen that for all BPF harmonics the calculated SPL emitted by the tower is higher than the one emitted by the rotor. The global maximum of the rotor-induced SPL is about $8 \mathrm{~dB}$ lower compared to the global peak of the tower-induced SPL; both occur at observer $\mathrm{C}$. The emission from the rotor shows a strong directivity to the upstream and downstream direction, with clearly lower amplitudes at observers B and D. At BPF, the emission of the tower shows the same directivity but is less pronounced, whereas the directional differences at higher harmonics of BPF are marginal. The SPL increase in the plane of rotation for frequencies below BPF is mainly caused by the tower emission. This is similar to the increase in amplitudes in the tower base load spectra for $M_{x}$ caused by pressure fluctuations on the tower surface, which is described in the previous section. Thus, SPL increase at frequencies be- 

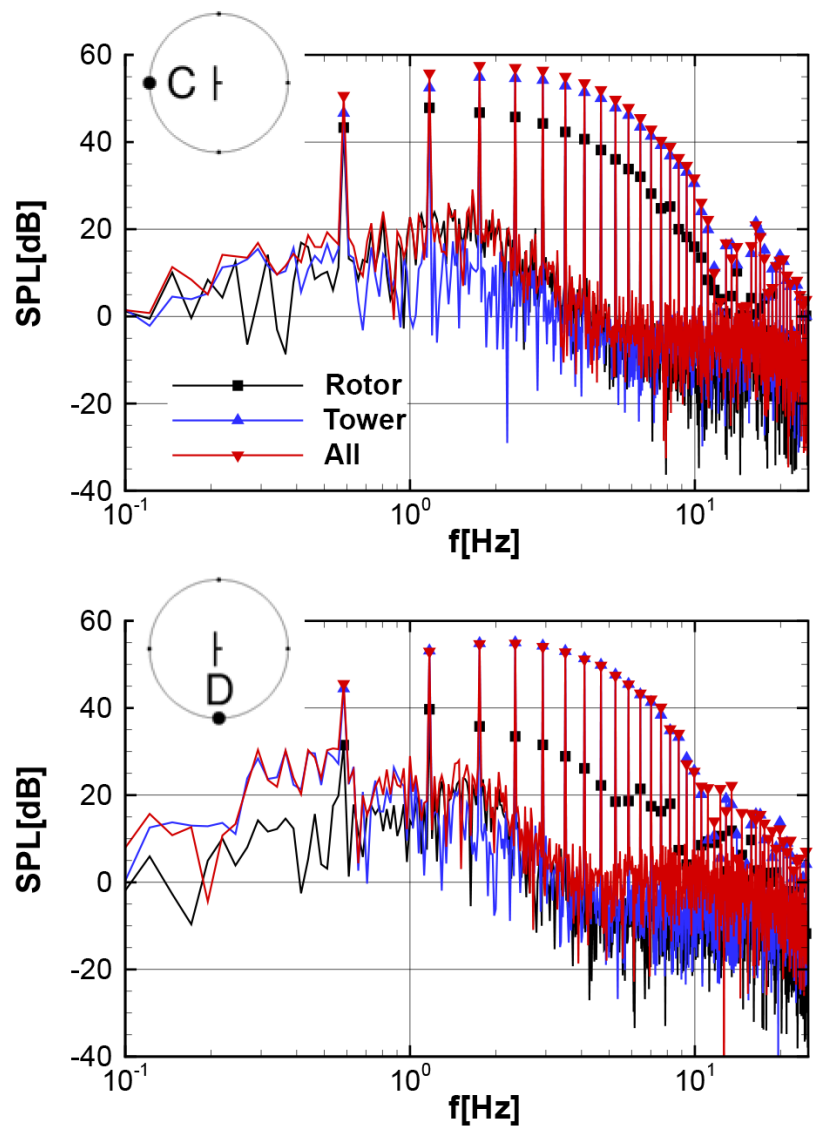

Figure 10. Spectra of unweighted SPL (reference sound pressure of $20 \mu \mathrm{Pa}$ ) at two observer positions on the ground with a distance of $1000 \mathrm{~m}$ from the turbine for case LC2_FSC1SD. Comparison of noise emitted from rotor, tower and all surfaces.

low BPF is very likely induced by surface pressure fluctuations due to vortex shedding at the tower, too. Looking at the noise carpet for the third BPF harmonic in Fig. 11 gives more insight into the directivity. The rotor emission is strongly directed towards 20 and $190^{\circ}$, whereas for the tower emission only a small shift in the generally concentric shape towards $220^{\circ}$ is present. The superposed signal shows a directivity towards $180 / 350^{\circ}$ and is slightly biased upstream. The result also shows that the shape of the SPL isolines beyond approximately the $500 \mathrm{~m}$ radius around the turbine is independent of the radius. The same behavior can be observed for the other harmonics of BPF. Thus, the previously regarded observers at the $1000 \mathrm{~m}$ radius are clearly out of near-field effects for BPF harmonics.

\subsection{Influence of degrees of freedom at uniform inflow}

In the second study, the cases LC2_FSC1SD, LC2_FSC1 and LC2_FSC3 are regarded. The aim is to evaluate the influence of the degrees of freedom of the structural model on the low-frequency emissions from the wind turbine. Case
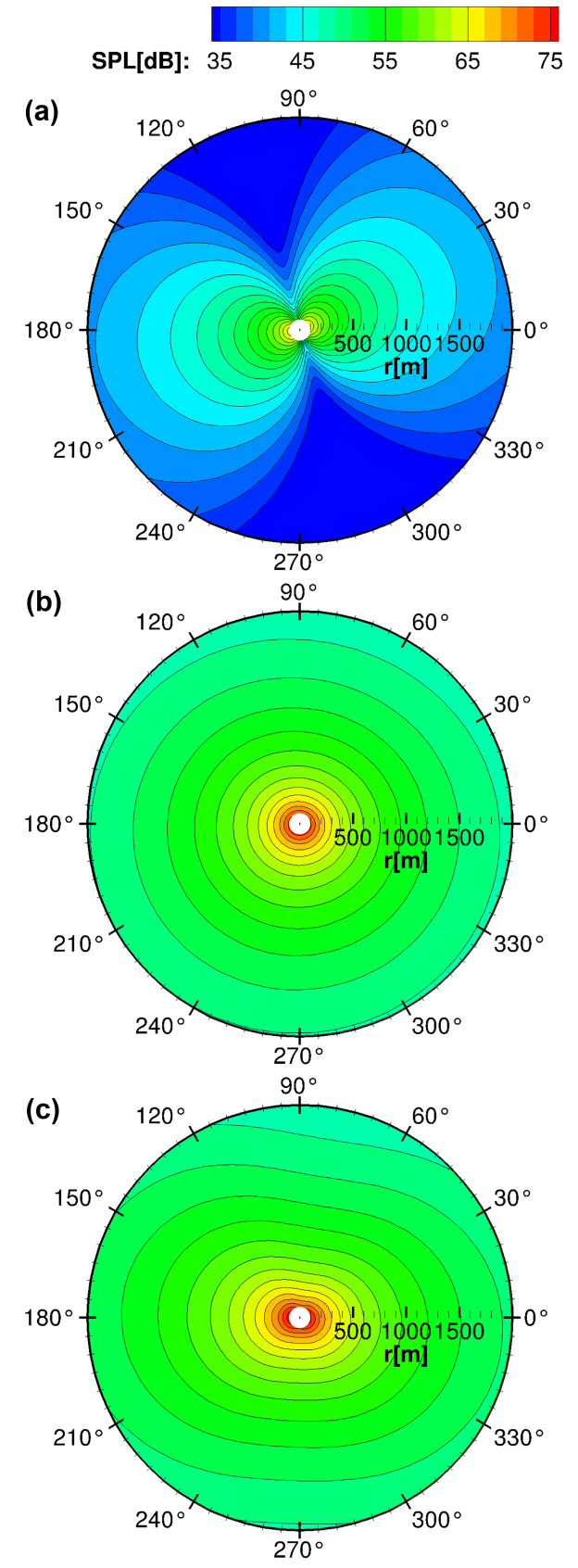

Figure 11. Unweighted SPL (reference sound pressure of $20 \mu \mathrm{Pa}$ ) at third BPF harmonic $(1.755 \mathrm{~Hz})$ on the ground around the turbine for case LC2_FSC1SD. Aeroacoustic emission from rotor (a), tower (b) and all surfaces (c). $\triangle$ SPL between black contour lines is $2 \mathrm{~dB}$.

LC2_FSC1SD has zero degrees of freedom but considers the mean blade deformation of case LC2_FSC1 where only the rotor blades are flexible; thus, it is chosen as reference case for this study. 

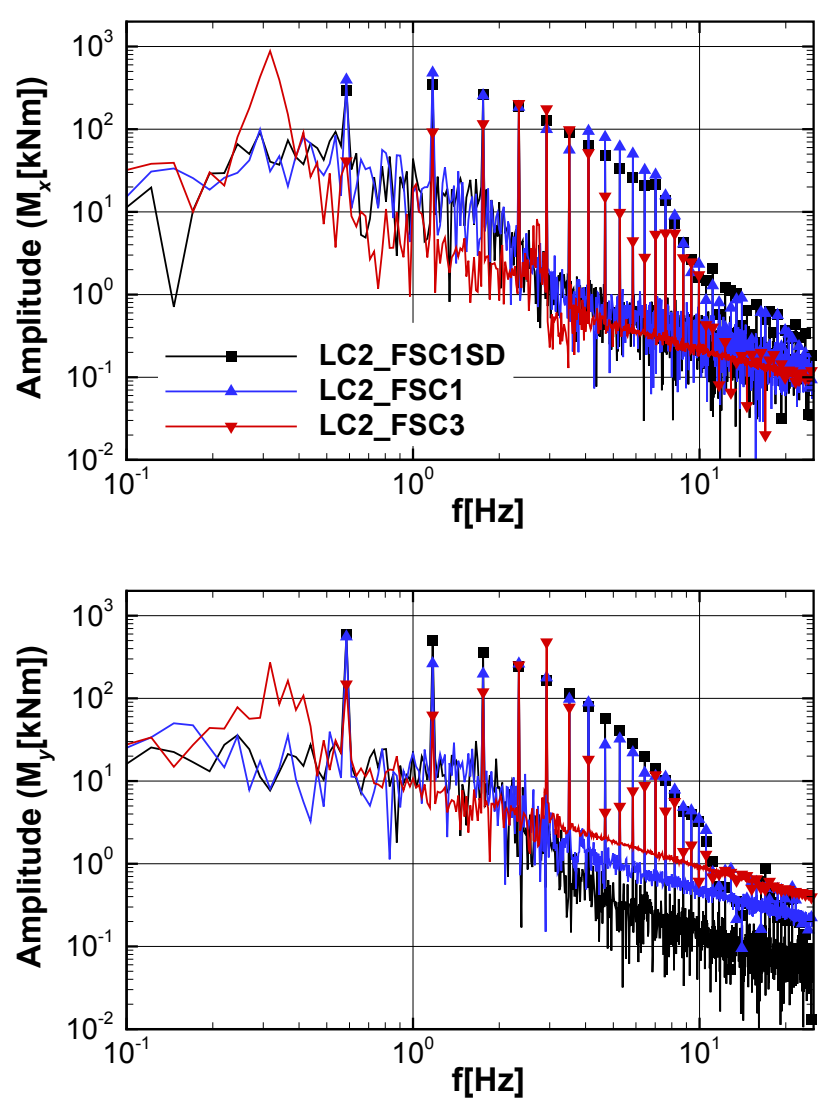

Figure 12. Spectra of tower base bending moments for the cases LC2_FSC1SD, LC2_FSC1 and LC2_FSC3.

\subsubsection{Tower base loads}

The spectra of the tower base loads for all three cases are plotted in Fig. 12. The flexibility of the rotor blades in case LC2_FSC1 mainly has an impact on the amplitudes at harmonics of BPF. $M_{x}$ amplitudes increase with the highest peaks at the first and second harmonic of BPF rising by more than $30 \%$ compared to case LC2_FSC1SD. By contrast, a decrease is observed for $M_{y}$, especially for the second and third harmonic of BPF.

There are two effects which go hand in hand, both having an influence on the tower base loads. When considering the flexibility of the blades, on the one hand, gravitational forces and inertial forces start acting and, on the other hand, aerodynamic forces change due to unsteady deflection of the blades. The mean blade tip deflection applied in case LC2_FSC1SD is $6.34 \mathrm{~m}$ out of plane (OOP) and $-0.58 \mathrm{~m}$ in plane (IP). In case LC2_FSC1 the OOP deflection reaches its maximum of approximately $6.46 \mathrm{~m}$ when the blade is passing the tower, just before the blade deformation is reduced due to the tower blockage. The IP deflection oscillates between -0.13 and $-1.02 \mathrm{~m}$, which is mainly caused by the gravitational force that makes the blade bend downwards. Due to the inertia of the blade, the IP blade tip velocity reaches its maximum just after the tower passage. This increases the absolute velocity of the blade when passing the tower and the relative flow velocity on the blade. On the other hand, the swinging of the blades mainly induces structural forces in $y$ and $z$ direction, which explains the increase in $M_{x}$ amplitudes at BPF.

The enabled flexibility of the tower in case LC2_FSC3 shows a much stronger impact on the tower base loads compared to case LC2_FSC1 as it significantly changes the structural eigenmodes of the turbine. In $M_{x}$ and $M_{y}$, the amplitudes at first, second and third harmonics of BPF are clearly reduced. Especially the reduction at BPF is remarkable: over $70 \%$ for both loads. For $M_{x}$ the amplitude at BPF even drops to the level of the broadband fluctuations of the other two cases. For $M_{y}$ the maximum amplitude shifts to the fifth harmonic of BPF, which is close to three structural eigenfrequencies of the turbine. For $M_{x}$ it occurs at approximately $0.32 \mathrm{~Hz}$, which matches the first side-side bending mode of the tower. An increase in the amplitudes in the frequency range around $0.32 \mathrm{~Hz}$ can also be observed for $M_{y}$ but is less pronounced. The first fore-aft bending mode is also at this frequency, but the aerodynamic damping is much higher compared to the side-side direction.

\subsubsection{Aeroacoustic emission}

The increase in degrees of freedom in the structural model only marginally influences the SPL at the observers. The spectrum at observer position $C$ shows a small decrease in the amplitude at BPF while there is a small increase at second to sixth harmonics of BPF. However, observer D shows a small increase at BPF while amplitudes of higher harmonics are almost unchanged. Generally, the effect is a bit stronger for case LC2_FSC3. These small changes might be an impact of the slightly reduced blade-tower distance and the increased blade tip velocity when the blade passes the tower, which is reported in the previous section. For frequencies below BPF, the maximum amplitude increases slightly, which could be induced by the structural eigenmodes of the turbine as well as by the impact of vortex shedding at the tower.

\subsection{Influence of inflow}

In the last study the influence of inflow conditions on the tower base loads and on the aeroacoustic emission is investigated. While uniform inflow is applied for the previous studies, more realistic inflow is considered in this study. Two cases - one with vertically sheared inflow (LC3_FSC3) and one with turbulent vertically sheared inflow (LC4_FSC3) are compared to the uniform inflow case (LC2_FSC3). For the turbulent inflow case, a longer time series is evaluated in order to obtain more representative results. 

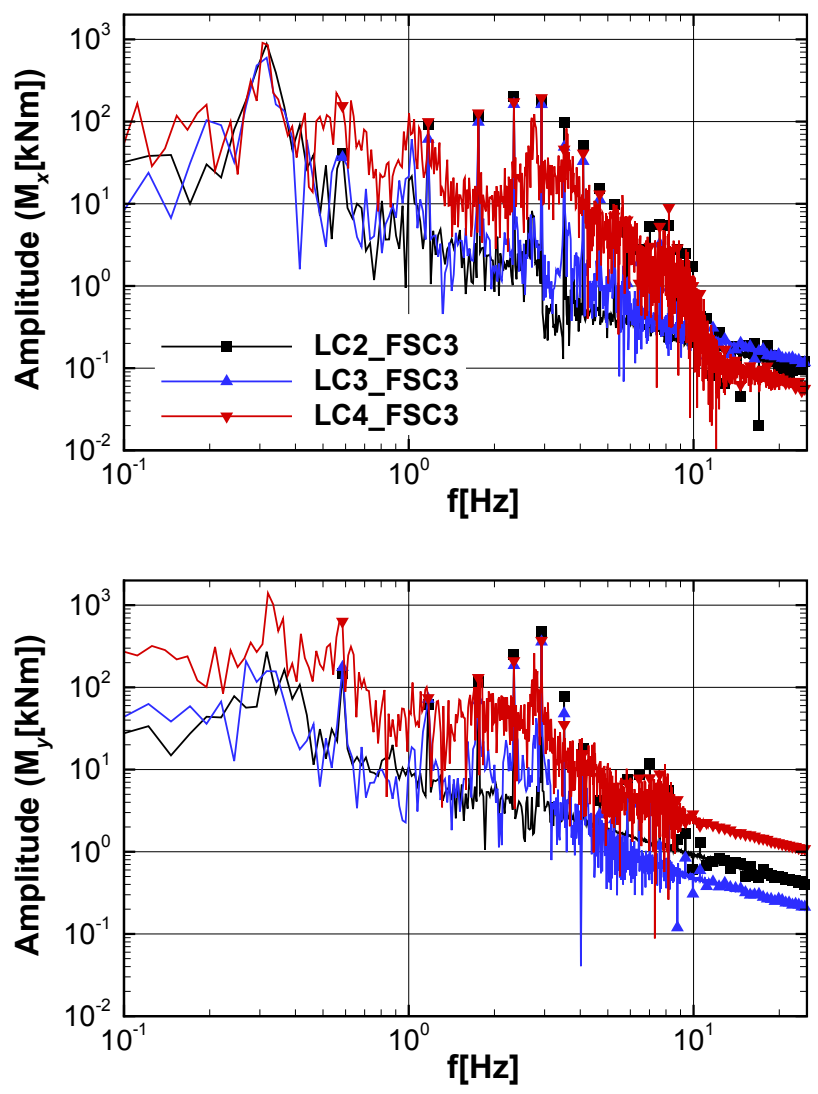

Figure 13. Spectra of tower base bending moments for the cases LC2_FSC3, LC3_FSC3 and LC4_FSC3.

\subsubsection{Tower base loads}

The spectra of tower base loads in Fig. 13 show that for case LC3_FSC3 an increase in amplitudes is only present for $M_{y}$ at BPF. Amplitudes at higher harmonics of BPF tend to reduce for $M_{x}$ and $M_{y}$. The result also shows that the broadband load level at frequencies between the first and fifth BPF harmonics rises. For $M_{x}$ there is a clear peak just above $1 \mathrm{~Hz}$, which even exceeds the peak at BPF. The reduction in amplitudes at higher harmonics of BPF can be explained as a result of the reduced inflow velocity below hub height due to the power law profile. Because of the lower aerodynamic thrust in this region, OOP deflection in front of the tower reduces to approximately 5.5 compared to $6.46 \mathrm{~m}$ in case LC2_FSC3. The rise of amplitudes at BPF can be explained as an effect of vertical shear. While blade passing is a short pulse and many higher harmonics of BPF are excited, the effect of vertical shear stretches over the whole revolution and is much closer to a sine function. Thus, the excitation of higher harmonics of BPF is much weaker compared to blade passing. The combination of vertical shear and reduced blade-passing effect finally leads to an increase in amplitudes at BPF while amplitudes at higher harmonics decrease.
By superimposing turbulence on the vertically sheared flow in case LC4_FSC3, the character of the spectra changes as the amplitudes at BPF harmonics become much less prominent. There are some clear peaks remaining, but the broadband load level massively increases. The global maximum now arises for $M_{y}$ at approximately $0.32 \mathrm{~Hz}$, corresponding to an eigenmode of the structural model. Additionally the amplitude at BPF is strongly increased for $M_{x}$ and $M_{y}$; however, side peaks occur that are partially even higher. The amplitude at approximately $1 \mathrm{~Hz}$ further increases compared to case LC3_FSC3, and another wide peak appears at frequencies around approximately $2.75 \mathrm{~Hz}$, which again corresponds to nearby structural eigenmodes. The higher amplitudes at frequencies near structural eigenmodes can be explained by the broadband excitation due to the influence of turbulent inflow on the aerodynamic loads. Without turbulent inflow the main excitation occurs at BPF harmonics because all unsteady effects except for the vortex shedding are periodic with BPF (blade-tower interaction, tilt angle, vertical shear).

\subsubsection{Aeroacoustic emissions}

Figure 14 shows the spectra of the acoustic spectra of the SPL at observers $C$ and D for the regarded cases. The vertically sheared inflow (case LC3_FSC3) leads to a slight decrease in SPL at BPF harmonics with a stronger effect at higher frequencies. Only a small increase in amplitude can be observed at BPF for observer D. For observer $\mathrm{C}$ an increase in the broadband noise level between approximately 2 and $10 \mathrm{~Hz}$ can be found, but it does not exceed $30 \mathrm{~dB}$. The reduction in SPL can be explained with the reduced blade tip deflection in front of the tower already mentioned above, which reduces the pressure fluctuations on the tower.

Taking the turbulent inflow into account (case LC4_FSC3) leads to an increase in the broadband noise level due to turbulent inflow noise, generated by the interaction of the rotor blade with the turbulence. The inflow noise is emitted from the rotor and predominantly directed in upstream and downstream direction, leading to higher broadband noise levels at observer C compared to observer D. Since the rotor blades encounter the turbulence at considerably higher relative velocity than the tower, the emission from the tower hardly increases compared to case LC3_FSC3. However, despite the increased broadband noise level, the peaks at BPF harmonics are still dominant at both observer positions.

\section{Discussion}

In the first study the influence of the presence of the tower and of steady blade deformation on low-frequency emissions is evaluated at uniform inflow conditions in stand-alone CFD simulations. Concerning the aerodynamic loads, the presence of the tower leads to an increase in amplitudes at BPF and its higher harmonics. Applying a steady deformation to the 

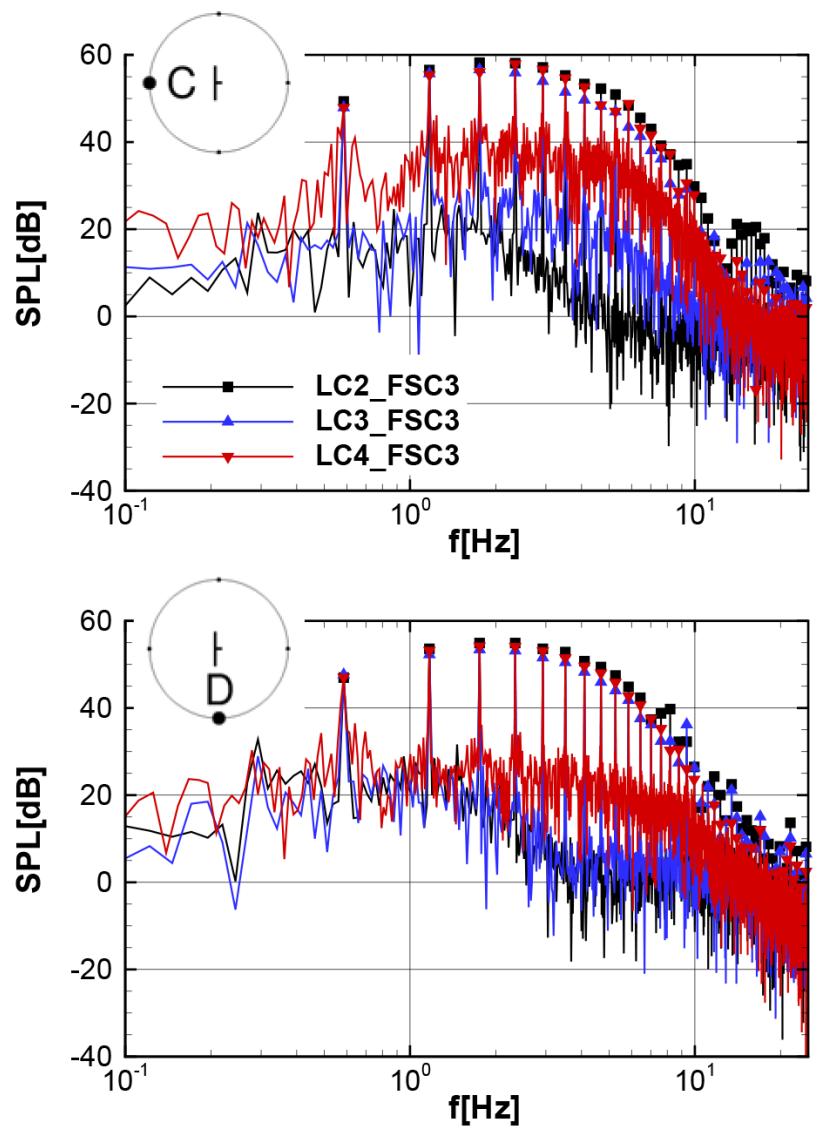

Figure 14. Spectra of unweighted SPL (reference sound pressure of $20 \mu \mathrm{Pa}$ ) at two observer positions on the ground with a distance of $1000 \mathrm{~m}$ from the turbine for cases LC2_FSC3, LC3_FSC3 and LC4_FSC3.

rotor blades further increases the amplitudes especially for higher harmonics due to the stronger blade-tower interaction. Splitting the loads up into rotor and tower loads shows that the major part of the fluctuations originates from the tower and is caused by blade-tower interaction. Load oscillations induced by vortex shedding can be observed but do not play an important role. Evaluating the SPL on the ground at a distance of $1000 \mathrm{~m}$ shows similar results. Through the presence of the tower a tonal noise emission with prominent peaks at BPF harmonics arises. Reduced blade-tower distance further increases the amplitudes of BPF harmonics especially at higher frequencies. Comparing the contributions of tower and rotor to the noise emission shows a strong directivity for the rotor emission in the direction of the rotor axis and a weak directivity for the tower emission except at BPF. Generally the emission from the tower is stronger in all directions in the regarded frequency range. This corresponds to the findings by Yauwenas et al. (2017), who did research on blade-passage noise and claimed a significant contribution of the tower. While Yauwenas et al. (2017) investigated a small model turbine with a symmetric blade in stationary air and a BPF of $45 \mathrm{~Hz}$, the present study shows that their assumption is also valid for a realistic multi-megawatt turbine under uniform inflow and a BPF in the low-frequency range.

In a second study, the influence of degrees of freedom in the structural model is investigated using three cases: one with steady blade deformation already regarded in the first study, another with flexible blades, and a third with additionally flexible tower and foundation. Flexible blades have only a minor impact on the calculated tower base loads. Structural eigenmodes play a more significant role in the third case when tower and foundation are flexible too. The peaks at BPF harmonics are still prominent, but the amplitudes change and the maxima are shifted towards BPF harmonics close to structural eigenfrequencies. Additionally, peaks corresponding to the first bending modes of the tower $(0.32 \mathrm{~Hz})$ occur, being dominant in the spectrum of $M_{x}$. Concerning aeroacoustics, the emission slightly increases but no clear influence of structural eigenmodes can be found in the regarded frequency range.

The third study deals with the influence of the inflow condition on the emissions. Uniform inflow is compared to vertically sheared inflow with and without turbulence. For vertical shear inflow, tower base loads tend to increase at BPF and decrease at higher harmonics of BPF. With superimposed turbulence, the peaks become much less prominent since the broadband load level rises. Amplitudes at frequencies close to structural eigenmodes rise, and BPF harmonics become less dominant in the spectra. The tonal noise level of the aeroacoustic emission tends to reduce slightly with the vertical shear and increase again due to the superimposed turbulence. The broadband noise level strongly increases, especially for observers upstream and downstream of the turbine, which is mainly caused by turbulent inflow noise emitted by the rotor. Thus, the BPF harmonics become less prominent but are still dominant in the spectra.

As a generic wind turbine is investigated, no measurements for validation are available. Nevertheless, a qualitative comparison between the presented results and two studies found in the literature is drawn. Zieger and Ritter (2018) showed seismic measurements in Germany that suggest an independence of discrete frequency peaks and blade-passing frequency. Although the amplitudes increase with increasing wind speed and rotational speed, the frequencies of the peaks do not change. This can be interpreted as a dominance of structural eigenmodes of the turbine in the origin of the seismic waves. However, at high (rated) rotational speed the dominant frequencies correspond very well to harmonics of the blade-passing frequency. Saccorotti et al. (2011) analyzed seismic measurements of a gravitational wave observatory in Italy close to a wind farm and found steady spectral lines as well as time-varying peaks which could all be identified as emitted by a wind turbine. The results of both studies coincide with the findings of the presented paper where tower base loads at BPF harmonics close to eigenfrequencies of the turbine are prominent in the spectra. The tonal char- 
acter of the low-frequency noise was also shown in acoustic field measurements (Hansen et al., 2017; Pilger and Ceranna, 2017). They showed that the BPF harmonics are dominant in the measured spectra and thus the peak frequencies shift depending on the rotational speed of the turbine. Pilger and Ceranna (2017) furthermore compared measurements of a single $200 \mathrm{~kW}$ turbine to estimated SPL from the Viterna method (Viterna, 1981). They found an underestimation of SPL which they explained with environmental conditions neglected in the model. Taking the present study into account, it is more likely that the neglect of tower emission in the Viterna method has a major impact on the results.

Despite the advanced modeling approach applied in the presented study, there are still several limitations that have to be mentioned. In the applied FW-H calculations, effects of unsteady flow field, refraction and reflection of acoustic waves and atmospheric layering are not taken into account for the propagation. On the other hand, this makes the method very suitable for the investigation of the aeroacoustic emission of the turbine, as the SPL at the observer positions is not influenced by the effects mentioned above. Due to the computationally expensive CFD approach, there are limitations concerning the length of the time series and temporal resolution and consequently the statistical convergence of the results and the resolved frequency range. Although the flexibility of rotor blades, tower and foundation is considered in the simulations, further degrees of freedom are neglected. The drive train is kept totally rigid and at fixed rotational speed. As SIMPACK is a MBS solver and only deformations of points along a beam are transferred, eigenmodes of the shell cannot be considered in the presented approach. However, the shortcomings mentioned do not change the general findings of this paper.

\section{Conclusions}

In the present paper the low-frequency emissions from a generic $5 \mathrm{MW}$ turbine are investigated using a high-fidelity time-resolved fluid-structure coupled CFD approach. Three different studies are conducted to identify sources, to better understand mechanisms and to evaluate the influence of the model complexity on the resulting emissions. Tower base loads are compared to study the effect of structure-borne noise as seismic wave propagation cannot be calculated with the presented method. The aeroacoustic noise propagation is computed using a Ffowcs-Williams-Hawkings method. To consider aeroelasticity in the simulations, a new coupling of the CFD solver FLOWer to the MBS solver SIMPACK is presented in this paper. With this method not only blade deformation can be taken into account, but deformations, translations and rotations of all parts of the turbine. Thus, fluidstructure coupled simulations with flexible tower and foundation could be conducted.
A major advantage compared to lower-fidelity approaches is that, as all geometries of the turbine are fully resolved, the unsteady pressure distributions on all surfaces, and thus all aerodynamic loads, are a direct outcome of the simulations. Regarding the aeroacoustic emission it is found that the blade-tower interaction plays a key role and the noise emitted from the tower is higher compared to the noise emitted from the rotor. Only an indirect impact of fluid-structure coupling on the aeroacoustics could be observed. Elastic blades reduce the distance between blade and tower and thus increase the strength of the blade-tower interaction. Turbulent inflow on the other side mainly influences the broadband noise level of the rotor. For the regarded turbulence level of $16 \%$ the noise has a tonal character with dominant peaks at blade-passing frequency harmonics.

Blade-tower interaction also has a great influence on the tower base loads; however, with increasing degrees of freedom structural eigenmodes play a much stronger role than for the aeroacoustic emission and amplitudes at eigenfrequencies become more dominant when turbulent inflow is applied. Nevertheless, blade-passing frequency harmonics can still be identified in the spectra. For aerodynamic load fluctuations at uniform inflow, it is found that the contribution of the tower exceeds the contribution of the rotor.

Several conclusions for the modeling of low-frequency emissions using CFD simulations can be drawn from the conducted studies. The blade-tower interaction is found to be the main source of aeroacoustic noise and triggers a major part of the aerodynamic load fluctuations. The tower itself as well as a realistic blade-tower distance has to be considered in the simulation to capture the blade-tower interaction properly. Fluid-structure coupling is the most appropriate way to a realistic blade-tower distance and is mandatory if structural emission shall be regarded. Moreover, the acoustic emission from the tower has to be considered in the noise evaluation and the loads on the tower have to be included in the fluidstructure coupling. Concerning the structural emission, it is not only the flexibility of the rotor blades but also that of the tower and foundation that have to be taken into account as they change the character of the tower base load spectra. Turbulent inflow should also be taken into account because it enhances the excitation of structural eigenmodes.

The findings can be transferred to any modeling method of low-frequency emissions from wind turbines. The method has to be capable of capturing the impact of blade passing not only on the blades but also on the tower and its effect, on the one hand, on the aerodynamic load fluctuations and, on the other hand, on the aeroacoustic noise emission.

Future work will deal with several of the listed limitations. A slightly smaller commercial wind turbine will be investigated numerically with the presented approach and field measurements will be available for comparison. Subsequently, the turbine will be simulated taking into account the operational conditions of the measurements. The influence of full shell coupling on the low-frequency emission 
will be investigated in a future study. Based on the presented findings, constructional measures such as lattice towers, increased blade tower distance or swept blades are likely to reduce low-frequency emissions and should be taken into account for future research.

Data availability. Data of the NREL 5 MW turbine are available from Jonkman et al. (2009).

Author contributions. LK implemented the coupling, performed the CFD-MBS simulations and wrote most of the paper. JG was responsible for the acoustic simulations and the turbulent inflow and contributed parts of the manuscript. FW contributed parts of the manuscript. TL and EK initiated the research, supervised the work and revised the manuscript.

Competing interests. The authors declare that they have no conflict of interest.

Acknowledgements. The studies were conducted as part of the joint research project "Objective Criteria for Seismic and Acoustic Emission of Inland Wind Turbines (TremAc), FKZ 0325839A", funded by the German Federal Ministry for Economic Affairs and Energy (BMWi). The authors are grateful for the financial support. The authors gratefully acknowledge the High Performance Computing Center Stuttgart for providing computational resources within the project WEALoads.

Edited by: Alessandro Bianchini

Reviewed by: three anonymous referees

\section{References}

Arnold, M., Cheng, P. W., and Biskup, F.: Simulation of FluidStructure-Interaction on Tidal Current Turbines Based on Coupled Multibody and CFD Methods, in: The Twenty-third International Offshore and Polar Engineering Conference, International Society of Offshore and Polar Engineers, available at: https: //www.onepetro.org/conference-paper/ISOPE-I-13-101 (last access: 9 October 2018), 2013.

Bekiropoulos, D., Lutz, T., Baltazar, J., Lehmkuhl, O., and Glodic, N.: D2013-3.1: Comparison of benchmark results from CFDSimulation, Deliverable report, KIC-OFFWINDTECH, 2013.

Bozorgi, A., Ghorbaniasl, G., and Nourbakhsh, S.: The reduction in low-frequency noise of horizontal-axis wind turbines by adjusting blade cone angle, Int. J. Environ. Sci. Te., 1-14, https://doi.org/10.1007/s13762-017-1639-x, 2018.

Ghasemian, M. and Nejat, A.: Aerodynamic noise prediction of a horizontal Axis wind turbine using improved delayed detached eddy simulation and acoustic analogy, Energ. Convers. Manage., 99, 210-220, https://doi.org/10.1016/j.enconman.2015.04.011, 2015.
Gortsas, T. V., Triantafyllidis, T., Chrisopoulos, S., and Polyzos, D.: Numerical modelling of micro-seismic and infrasound noise radiated by a wind turbine, Soil Dyn. Earthq. Eng., 99, 108-123, https://doi.org/10.1016/j.soildyn.2017.05.001, 2017.

Hansen, K. L., Zajamšek, B., and Hansen, C. H.: The Occurrence of Nocturnal Wind Farm Rumbling Noise, 7th International Conference on Wind Turbine Noise Rotterdam, 2-5 May 2017, Willem Burger Complex, De Doelen, Rotterdam, Netherlands, 1-11, 2017.

Illg, J., Lutz, T., and Krämer, E.: Aeroacoustic Simulation of an Airfoil in Turbulent Inflow, in: 6th International Conference on Wind Turbine Noise, Glasgow, 20-23 April 2015, Radisson Blu Hotel, 301 Argyle Street, Glasgow G2 8DL, UK, 2015.

Jassmann, U., Berroth, J., Matzke, D., Schelenz, R., Reiter, M., Jacobs, G., and Abel, D.: Model predictive control of a wind turbine modelled in Simpack, J. Phys.-Conference Series, IOP Publishing, 524, 1-11, https://doi.org/10.1088/17426596/524/1/012047, 2014.

Jonkman, J., Butterfield, S., Musial, W., and Scott, G.: Definition of a 5-MW reference wind turbine for offshore system development, Tech. rep., National Renew. Energ. Lab.(NREL), Golden, CO, United States, https://doi.org/10.2172/947422, 2009.

Knopper, L. D., Ollson, C. A., McCallum, L. C., Whitfield Aslund, M. L., Berger, R. G., Souweine, K., and McDaniel, M.: Wind Turbines and Human Health, Frontiers in Public Health, 2, 63, https://doi.org/10.3389/fpubh.2014.00063, 2014.

Kowarsch, U., Keßler, M., and Krämer, E.: High order CFDsimulation of the rotor-fuselage interaction, 39th European Rotorcraft Forum, 3-9 September, Moscow, 2013.

Kranzinger, P. P., Kowarsch, U., Schuff, M., Keßler, M., and Krämer, E.: Advances in parallelization and high-fidelity simulation of helicopter phenomena, in: High Performance Computing in Science and Engineering, Springer International Publishing, 15, 479-494, https://doi.org/10.1007/978-3-319-246338_31, 2016.

Kroll, N., Rossow, C.-C., Becker, K., and Thiele, F.: The MEGAFLOW project, Aerosp. Sci. Technol., 4, 223-237, https://doi.org/10.1016/S1270-9638(00)00131-0, 2000.

Li, Y., Castro, A., Martin, J., Sinokrot, T., Prescott, W., and Carrica, P.: Coupled computational fluid dynamics/multibody dynamics method for wind turbine aero-servo-elastic simulation including drivetrain dynamics, Renew. Energ., 101, 1037-1051, https://doi.org/10.1016/j.renene.2016.09.070, 2017.

Liu, W.: A review on wind turbine noise mechanism and de-noising techniques, Renew. Energ., 108, 311-320, https://doi.org/10.1016/j.renene.2017.02.034, 2017.

Luhmann, B., Seyedin, H., and Cheng, P.-W.: Aero-structural dynamics of a flexible hub connection for load reduction on two-bladed wind turbines, Wind Energy, 20, 521-535, https://doi.org/10.1002/we.2020, 2017.

Lutz, T., Arnold, B., Bekiropoulos, D., Illg, J., Krämer, E., Wolf, A., Hann, R., and Kamruzzaman, M.: Prediction of Flow-Induced Noise Sources of Wind Turbines and Application Examples, Int. J. Aeroacoust., 14, 675-714, https://doi.org/10.1260/1475472X.14.5-6.675, 2015.

Madsen, H. A.: Low frequency noise from wind turbines mechanisms of generation and its modelling, J. Low Freq. Noise Vib., 29, 239-251, https://doi.org/10.1260/0263-0923.29.4.239, 2010. 
Mann, J.: The spatial structure of neutral atmospheric surface-layer turbulence, J. Fluid Mech., 273, 141-168, https://doi.org/10.1017/S0022112094001886, 1994.

Matha, D., Hauptmann, S., Hecquet, T., and Kühn, M.: Methodology and results of loads analysis of wind turbines with advanced aeroelastic multi-body simulation, DEWEK, Bremen, 2010.

Menter, F. R.: Two-equation eddy-viscosity turbulence models for engineering applications, AIAA Journal, 32, 1598-1605, https://doi.org/10.2514/3.12149, 1994.

Pilger, C. and Ceranna, L.: The influence of periodic wind turbine noise on infrasound array measurements, J. Sound Vib., 388, 188-200, https://doi.org/10.1016/j.jsv.2016.10.027, 2017.

Saccorotti, G., Piccinini, D., Cauchie, L., and Fiori, I.: Seismic noise by wind farms: a case study from the Virgo Gravitational Wave Observatory, Italy, B. Seismol. Soc. Am., 101, 568-578, https://doi.org/10.1785/0120100203, 2011.

Sayed, M., Lutz, T., and Krämer, E.: Aerodynamic investigation of flow over a multi-megawatt slender bladed horizontal-axis wind turbine, in: Renewable Energies Offshore, CRC Press, 773-780, ISBN 9781138028715, 2015.

Sayed, M., Lutz, T., Krämer, E., Shayegan, S., Ghantasala, A., Wüchner, R., and Bletzinger, K.-U.: High fidelity CFD-CSD aeroelastic analysis of slender bladed horizontalaxis wind turbine, J. Phys. Conf. Ser., 753, 042009, https://doi.org/10.1088/1742-6596/753/4/042009, 2016.

Schepers, J., Ceyhan, O., Boorsma, K., Gonzalez, A., Munduate, X., Pires, O., Sørensen, N., Ferreira, C., Sieros, G., Madsen, J., Voutsinas, S., Lutz, T., Barakos, G., Colonia, S., Heißelmann, H., Meng, F., and Croce, A.: Latest results from the EU project AVATAR: Aerodynamic modelling of $10 \mathrm{MW}$ wind turbines, J. Phys. Conf. Ser., 753, 022017, https://doi.org/10.1088/17426596/753/2/022017, 2016.

Schuff, M., Kranzinger, P., Keßler, M., and Krämer, E.: Advanced CFD-CSD coupling: Generalized, high performant, radial basis function based volume mesh deformation algorithm for structured, unstructured and overlapping meshes, in: Proceedings of the 40th European Rotorcraft Forum, Southhampton, Great Britain, 2014.

Schulz, C., Klein, L., Weihing, P., and Lutz, T.: Investigations into the Interaction of a Wind Turbine with Atmospheric Turbulence in Complex Terrain, J. Phys. Conf. Ser., 753, 032016, https://doi.org/10.1088/1742-6596/753/3/032016, 2016a.

Schulz, C., Meister, K., Lutz, T., and Krämer, E.: Investigations on the wake development of the MEXICO rotor considering different inflow conditions, in: New Results in Numerical and Experimental Fluid Mechanics X, Springer, 871-882, https://doi.org/10.1007/978-3-319-27279-5_76, 2016b.
Stammler, K. and Ceranna, L.: Influence of wind turbines on seismic records of the Gräfenberg array, Seismol. Res. Lett., 87, 1075-1081, https://doi.org/10.1785/0220160049, 2016.

Streiner, S., Hauptmann, S., Kühn, M., and Krämer, E.: Coupled fluid-structure simulations of a wind turbine rotor, in: Deutsche Windenergie-Konferenz (DEWEK), Bremen, Germany, DEWIGerman Wind Energy Institute, 2008.

Styles, P., Stimpson, I., Toon, S., England, R., and Wright, M.: Microseismic and infrasound monitoring of low frequency noise and vibrations from windfarms, Recommendations on the Siting of Windfarms in the Vicinity of Eskdalemuir, Scotland, Report to MOD/FTI/BWEA, 125 pp., available at: https://www.keele.ac.uk/geophysics/appliedseismology/ wind/Final_Report.pdf (last access: 9 October 2018), 2005.

Turnbull, C., Turner, J., and Walsh, D.: Measurement and level of infrasound from wind farms and other sources, Acoust. Aust., 40, 45-50, 2012.

Van den Berg, G.: The beat is getting stronger: the effect of atmospheric stability on low frequency modulated sound of wind turbines, J. Low Freq. Noise Vib., 24, 1-23, https://doi.org/10.1260/0263092054037702, 2005.

Viterna, L. A.: The NASA-LERC wind turbine noise prediction code, NASA CP, 2185, available at: https://ntrs.nasa.gov/search. jsp?R=19820015854 (last access: 9 October 2018), 1981.

Weihing, P., Letzgus, J., Bangga, G., Lutz, T., and Krämer, E.: Hybrid RANS/LES Capabilities of the Flow Solver FLOWer - Application to Flow Around Wind Turbines, in: Progress in Hybrid RANS-LES Modelling, Springer International Publishing, 369380, https://doi.org/10.1007/978-3-319-70031-1_31, 2018.

Yauwenas, Y., Zajamšek, B., Reizes, J., Timchenko, V., and Doolan, C. J.: Numerical simulation of blade-passage noise, The Journal of the Acoustical Society of America, 142, 1575-1586, https://doi.org/10.1121/1.5003651, 2017.

Zajamšek, B., Hansen, K. L., Doolan, C. J., and Hansen, C. H.: Characterisation of wind farm infrasound and low-frequency noise, J. Sound Vib., 370, 176-190, https://doi.org/10.1016/j.jsv.2016.02.001, 2016.

Zieger, T. and Ritter, J. R.: Influence of wind turbines on seismic stations in the upper rhine graben, SW Germany, J. Seismol., 22, 105-122, https://doi.org/10.1007/s10950-017-9694-9, 2018. 